\title{
Enantioseparation on Fluoro-Methylphenylcarbamates of Cellulose and Amylose as Chiral Stationary Phases for High-Performance Liquid Chromatography
}

\author{
Eiji Yashima, Chiyo Yamamoto, and Yoshio Окамото* \\ Department of Applied Chemistry, School of Engineering, \\ Nagoya University, Chikusa-ku, Nagoya 464-01, Japan
}

(Received February 24, 1995)

\begin{abstract}
Six phenylcarbamate derivatives of cellulose and amylose having both an electron-withdrawing fluoro group and an electron-donating methyl group on the phenyl moieties were synthesized, and their chiral recognition abilities were evaluated as chiral stationary phases for high-performance liquid chromatography. The resolving abilities were compared with those of chloro-methylphenylcarbamate derivatives of cellulose and amylose. The chiral recognition abilities of fluoro-methylphenylcarbamates of cellulose and amylose were dependent on the position of the substituents. The cellulose derivatives with fluoro and methyl groups at the meta and para positions showed higher resolving power than the ortho- and meta-disubstituted derivative as observed for the chloro-methylphenylcarbamate derivatives of cellulose. On the other hand, the ortho-substituted derivative of amylose, tris(5-fluoro-2-methylphenylcarbamate), showed high chiral recognition as well as the meta- and para-disubstituted derivatives. The different chiral recognition was discussed on the basis of IR and CD spectroscopic data.
\end{abstract}

KEY WORDS Enantiomer/Resolution / Phenylcarbamate / Polysaccharide / Cellulose / Amylose / Chiral Recognition / HPLC / Chiral Stationary Phase /

Enantiomers of biologically active compounds such as pharmaceuticals and agrochemicals often show quite different physiological behaviors in living systems. Therefore, detailed investigation on pharmacokinetics and physiological activities of both enantiomers should be done before use. Chromatographic enantioseparations, particularly, resolution by highperformance liquid chromatography (HPLC), has advanced considerably in the past decade and has become a practically useful method not only for determining their optical purity but also for obtaining optical isomers. Development of effective chiral stationary phases (CSPs) is the key in this method. Therefore, many CSPs for HPLC have been prepared. ${ }^{1-4}$ Among them, the CSPs consisting of phenylcarbamate derivatives of polysaccharides such as cellulose and amylose appear to be one of the most useful CSPs. ${ }^{5-7}$ In particular, 3,5dimethyl- or 3,5-dichlorophenylcarbamate derivatives of cellulose and amylose show high chiral recognition. ${ }^{8}$ Recently, we found that disubstituted phenylcarbamate derivatives having both an electron-withdrawing chloro group and an electron-donating methyl group on the phenyl moieties such as 3-chloro-4methyl- and 4-chloro-3-methylphenylcarbamates of cellulose and 5-chloro-2-methylphenylcarbamate of amylose also showed high chiral recognition. Effects of the position of substituents on the chiral recognition abilities of the phenylcarbamate derivatives were discussed using IR, ${ }^{1} \mathrm{H}$ NMR, and CD spectroscopic data. ${ }^{9-11}$

In this study, three kinds of disubstituted

\footnotetext{
* To whom all correspondence should be addressed.
} 


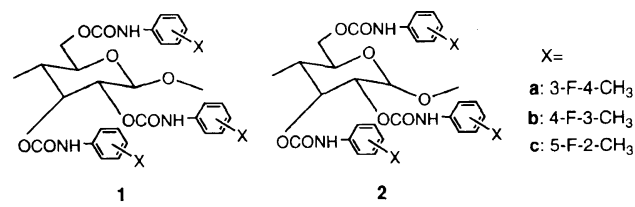

Figure 1. Structures of CSPs.

phenylcarbamates of cellulose and amylose having 3-fluoro-4-methyl (a), 4-fluoro-3-methyl (b), and 5-fluoro-2-methyl (c) substituents on the phenyl moieties (Figure 1) were prepared and their chiral recognition abilities as CSPs were evaluated by comparing with those for the corresponding chloro-methylphenylcarbamates.

\section{EXPERIMENTAL}

\section{Chemicals}

Cellulose (Avicel, DP 100) was purchased from Merck. Amylose (DP 60) was a gift from Nakano Vinegar (Handa, Japan). 3Fluoro-4-methylaniline and 2-fluoro-5-nitrotoluene were obtained from Aldrich. 4-Fluoro2-nitrotoluene, triphosgene, and (3-aminopropyl)triethoxysilane were of guaranteed reagent grade from Tokyo Kasei. Porous spherical silica gel with a mean particle size of $7 \mu \mathrm{m}$ and a mean pore diameter of $100 \mathrm{~nm}$ (Daiso gel SP-1000) was kindly supplied by Daiso Chemical. All solvents used in the preparation of CSPs were of analytical reagent grade, carefully dried, and distilled before use. Solvents used in the chromatographic experiments were of HPLC grade. Racemates (3-12) (Figure 2) were commercially available or were prepared by the usual method. ${ }^{12}$

\section{Synthesis of Tris(fluoro-methylphenylcarba- mate)s of Cellulose and Amylose}

4-Fluoro-3-methylaniline and 5-fluoro-2methylaniline were prepared by reduction of the corresponding fluoro-nitrotoluene using $\mathrm{SnCl}_{2} \cdot 2 \mathrm{H}_{2} \mathrm{O}$ and hydrochloric acid $(35 \%)$ in ethanol. Fluoro-methylphenyl isocyanates were prepared from the corresponding aniline

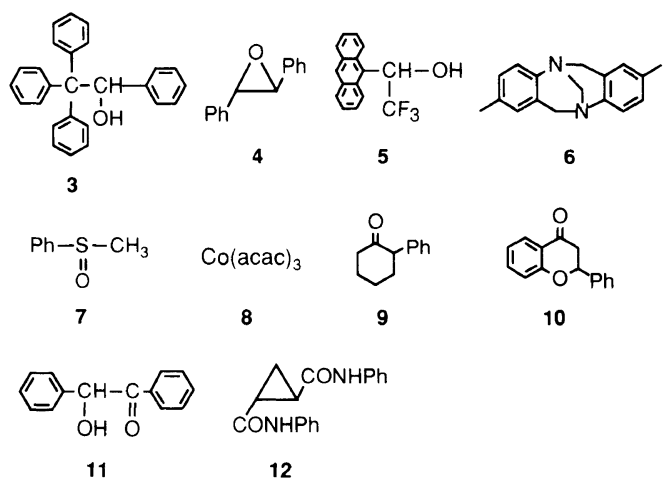

Figure 2. Structures of racemates.

Table I. Elemental analysis of phenylcarbamate derivatives of cellulose and amylose

\begin{tabular}{|c|c|c|c|}
\hline & $\mathrm{C} \%$ & $\mathrm{H} \%$ & N\% \\
\hline 1a & 58.37 & 4.39 & 5.25 \\
\hline $\mathbf{1 b}$ & 57.36 & 4.33 & 6.43 \\
\hline $1 c$ & 58.15 & 4.30 & 6.90 \\
\hline $\mathbf{2 a}$ & 58.13 & 4.36 & 5.32 \\
\hline 2b & 59.17 & 4.35 & 6.75 \\
\hline $2 c$ & 57.80 & 4.09 & 6.99 \\
\hline Calculated value ${ }^{\mathbf{a}}$ & 58.54 & 4.58 & 6.83 \\
\hline
\end{tabular}

${ }^{\text {a }}$ Estimated based on a repeated glucose unit.

derivatives by the conventional method using triphosgene in toluene at $c a .80^{\circ} \mathrm{C}$, and purified by distillation; 3-fluoro-4-methylphenyl isocyanate (bp $82^{\circ} \mathrm{C} / 27 \mathrm{mmHg}$ ), 4-fluoro-3-methylphenyl isocyanate (bp $65^{\circ} \mathrm{C} / 10 \mathrm{mmHg}$ ), and 5-fluoro-2-methylphenyl isocyanate (bp $85^{\circ} \mathrm{C} / 28 \mathrm{mmHg}$ ).

Phenylcarbamate derivatives of cellulose and amylose were prepared by the reaction of cellulose or amylose with an excess of the corresponding fluoro-methylphenyl isocyanate in dry pyridine at $c a .80^{\circ} \mathrm{C}^{8}$ The phenylcarbamates obtained were isolated as methanolinsoluble fractions or methanol- $\mathrm{H}_{2} \mathrm{O}(5: 1)$ insoluble fractions. Elemental analysis (Table I) and ${ }^{1} \mathrm{H}$ NMR data showed that hydroxy groups of cellulose and amylose were almost quantitatively converted into the carbamate moieties. 


\section{Preparation of Stationary Phase}

Each column packing material was prepared using macroporous silica gel as described previously and was packed into a stainless-steel tube $(25 \mathrm{~cm} \times 0.46 \mathrm{~cm}$ i.d. $)$ by conventional high-pressure slurry packing technique using a model CCP-085 Econo packer pump (Chemco). The plate numbers of the columns were 4500-6500 for benzene with hexane-2propanol $(90: 10)$ as the eluent at a flow rate of $0.5 \mathrm{ml} \mathrm{min}^{-1}$. 1,3,5-Tri-tert-butylbenzene was used as a non-retained compound for estimating the dead time $\left(t_{0}\right)$.

\section{Apparatus}

Chromatographic experiments were performed on a JASCO 880-PU chromatograph equipped with a UV (JASCO 875-UV) and a polarimetric (JASCO DIP-182) detectors at room temperature. A solution of a racemate $(1-10 \mu \mathrm{l})$ was injected into the chromatographic system with a Rheodyne Model 7125 injector. IR spectra were measured on a JASCO Fourier transform IR-7000 spectrometer as a $\mathrm{KBr}$ pellet. Circular dichroism (CD) spectra of polysaccharide derivatives were taken in THF solution (1.6 $\mathrm{mM}$ based on glucose units) on a JASCO J-720 L apparatus with a $0.1 \mathrm{~mm}$ optical cell at ambient temperature. UV spectra were recorded on a JASCO Ubest-55 spectrophotometer. ${ }^{1} \mathrm{H}$ NMR spectra were taken in pyridine- $d_{5}$ at $80^{\circ} \mathrm{C}$ with a Varian VXR-500S NMR spectrometer $(500 \mathrm{MHz})$ using tetramethylsilane (TMS) as the internal standard.

\section{RESULTS AND DISCUSSION}

Figure 3 shows a chromatogram of the resolution of racemic 1,2,2,2-tetraphenylethanol (3) on a column packed with amylose tris(4-fluoro-3-methylphenylcarbamate) (2b). The enantiomers eluted at retention times of $t_{1}$ and $t_{2}$ showing complete separation. Capacity factors, $k_{1}{ }^{\prime}\left[=\left(t_{1}-t_{0}\right) / t_{0}\right]$ and $k_{2}{ }^{\prime}$ $\left[=\left(t_{2}-t_{0}\right) / t_{0}\right]$, were 1.14 and 2.67 , respectively. Separation factor $\alpha\left[=k_{2}{ }^{\prime} / k_{1}{ }^{\prime}\right]$ and

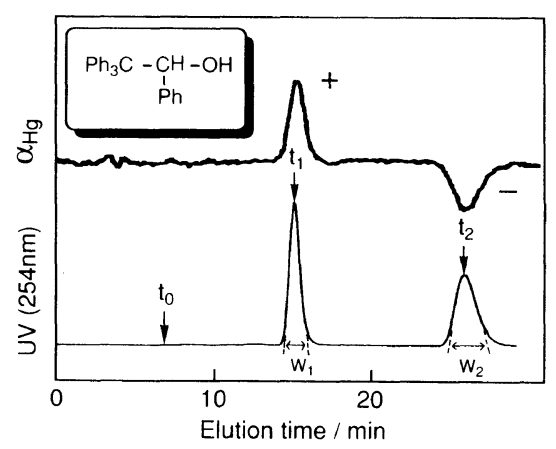

Figure 3. Separation of enantiomers of 1,2,2,2-tetraphenylethanol (3) on $\mathbf{2 b}$. Chromatographic conditions are shown in Table II.

resolution factor $R_{\mathrm{s}}\left[=2\left(t_{2}-t_{1}\right) /\left(\dot{w}_{1}+w_{2}\right)\right]$ were found to be 2.34 and 5.34 , respectively.

The results of the enantioseparation of ten racemates 3, trans-stilbene oxide (4), 1-(9anthryl)-2,2,2-trifluoroethanol (5), Tröger base (6), methyl phenyl sulfoxide (7), cobalt(III) tris(acetylacetonate) (8), 2-phenylcyclohexanone (9), flavanone (10), benzoin (11), and trans-cyclopropanedicarboxylic acid dianilide (12) on the fluoro-methylphenylcarbamates of cellulose and amylose are given in Table II. Meta- and para-disubstituted cellulose derivatives 1a and 1b showed higher resolving power than ortho- and meta-disubstituted derivative 1c and could resolve most of the racemates tested in the present study. On the other hand, amylose derivative $2 \mathrm{c}$ having a substituent at the ortho position also showed characteristic high chiral recognition comparable to the meta- and para-disubstituted derivatives $\mathbf{2 a}$ and $\mathbf{2 b}$. The elution order of most racemates was not influenced by the position of the substituents introduced on the phenyl groups of the cellulose or amylose derivatives. However, the elution order of several racemates 5-8, 10-12 on the cellulose derivatives was reversed compared with that on the corresponding amylose derivatives. These results indicate that the chiral recognition mechanism on the cellulose derivatives may be different from that of the amylose derivatives.

Similar results have been obtained on 
Table II. Resolution of $\mathbf{3}-\mathbf{1 2}$ on fluoro-methylphenylcarbamate derivatives ${ }^{\mathbf{a}}$

\begin{tabular}{|c|c|c|c|c|c|c|c|c|c|}
\hline & \multicolumn{3}{|c|}{$1 \mathbf{a}$} & \multicolumn{3}{|c|}{$1 b$} & \multicolumn{3}{|c|}{ 1c } \\
\hline & $k_{1}{ }^{\prime}$ & $\alpha$ & $R_{\mathrm{S}}$ & $k_{1}^{\prime}$ & $\alpha$ & $R_{\mathrm{S}}$ & $k_{1}^{\prime}$ & $\alpha$ & $R_{\mathrm{s}}$ \\
\hline 3 & $0.82(+)$ & 1.41 & 3.04 & $0.82(+)$ & 1.88 & 3.93 & $0.74(+)$ & $\sim 1$ & \\
\hline 4 & $0.42(+)$ & 1.79 & 3.79 & $0.37(+)$ & 1.81 & 3.00 & $0.48(+)$ & 1.55 & 2.35 \\
\hline 5 & $0.68(-)$ & 1.35 & 2.14 & $0.66(-)$ & 1.31 & 1.47 & $0.95(-)$ & 1.23 & 0.78 \\
\hline 6 & $0.76(+)$ & 1.41 & 2.97 & $0.73(+)$ & 1.15 & 0.87 & $0.61(+)$ & $\sim 1$ & \\
\hline 7 & $8.73(+)$ & 1.11 & 1.93 & $7.28(+)$ & 1.06 & 0.79 & $8.72(-)$ & $\sim 1$ & \\
\hline 8 & $1.14(+)$ & 1.83 & 4.73 & $0.96(+)$ & 1.40 & 2.10 & $0.83(+)$ & $\sim 1$ & \\
\hline 9 & $1.46(-)$ & 1.25 & 2.91 & $1.04(-)$ & 1.18 & 1.34 & $1.83(-)$ & 1.05 & \\
\hline 10 & $1.59 .(-)$ & 1.13 & 1.58 & $1.48(-)$ & 1.11 & 0.92 & $1.97(-)$ & 1.13 & 1.06 \\
\hline 11 & $3.67(-)$ & 1.14 & 1.91 & $3.56(-)$ & 1.24 & 2.51 & $4.62(+)$ & 1.10 & 0.99 \\
\hline \multirow[t]{3}{*}{12} & $1.40(-)$ & $\sim$ & & $1.47(-)$ & 1.29 & 1.13 & $1.04(-)$ & 1.34 & \\
\hline & \multicolumn{3}{|c|}{$\mathbf{2 a}$} & \multicolumn{3}{|c|}{$\mathbf{2 b}$} & \multicolumn{3}{|c|}{$2 c$} \\
\hline & $k_{1}{ }^{\prime}$ & $\alpha$ & $R_{\mathrm{S}}$ & $k_{1}{ }^{\prime}$ & $\alpha$ & $R_{\mathrm{s}}$ & $k_{1}{ }^{\prime}$ & $\alpha$ & $R_{\mathrm{S}}$ \\
\hline 3 & $0.98(+)$ & 2.49 & 5.79 & $1.14(+)$ & 2.34 & 5.34 & $0.98(+)$ & 1.64 & 0.74 \\
\hline 4 & $0.44(+)$ & 1.73 & 3.29 & $0.55(+)$ & 1.82 & 4.08 & $0.64(+)$ & 1.89 & 3.79 \\
\hline 5 & $0.54(+)$ & 1.12 & & $0.75(+)$ & 1.09 & & $0.94(+)$ & 1.15 & \\
\hline 6 & $0.81(+)$ & 1.23 & 1.50 & $0.86(+)$ & 1.40 & 2.32 & $0.97(-)$ & $\sim 1$ & \\
\hline 7 & $6.04(-)$ & 1.09 & 1.17 & $6.45(-)$ & 1.08 & & $7.13(-)$ & 1.14 & \\
\hline 8 & $0.97(-)$ & 1.11 & & $1.03(-)$ & $\sim 1$ & & $1.82(+)$ & $\sim 1$ & \\
\hline 9 & $1.24(-)$ & $\sim 1$ & & $1.41(-)$ & 1.08 & & $1.76(-)$ & 1.19 & 1.57 \\
\hline 10 & $1.44(+)$ & 1.36 & 2.83 & $1.92(+)$ & 1.13 & & $0.62(+)$ & 1.24 & \\
\hline 11 & $4.23(+)$ & 1.03 & & $5.58(+)$ & 1.17 & 2.05 & $.5 .01(+)$ & 1.60 & 4.10 \\
\hline 12 & $0.97(+)$ & 1.31 & 1.07 & $2.77(+)$ & $\sim 1$ & & $0.87(+)$ & 2.03 & 0.77 \\
\hline
\end{tabular}

${ }^{a}$ Eluent, hexane-2-propanol $(90: 10, \mathrm{v} / \mathrm{v})$; flow rate, $0.5 \mathrm{ml} \mathrm{min}{ }^{-1}$. The signs in parentheses represent the optical rotation of the first-eluted enantiomer.

chloro-methylphenylcarbamates of cellulose and amylose ${ }^{9-11} ; 3$-chloro-4-methyl and 4chloro-3-methyl derivatives of cellulose showed higher resolving power than the other orthosubstituted derivatives, while 5-chloro-2-methylphenylcarbamate exhibited the most effective chiral recognition ability in the amylose derivatives and resolved the all racemates $3-12$ with high $\alpha$ values. No reverse elution order of the enantiomers was observed on changing the chloro group to the fluoro group except for a few cases.

The fluoro-methylphenylcarbamates of cellulose and amylose are soluble in chloroform whereas the chloro-methylphenylcarbamates are insoluble. This higher solubility of the fluorine-containing derivatives will be useful for studying the chiral recognition mechanism by NMR spectroscopy. ${ }^{13} \mathrm{~A}$ detailed study is in progress.

It has been demonstrated that the most important adsorbing site for chiral recognition on the phenylcarbamate derivatives of polysaccharides is the polar carbamate residue, which can interact with enantiomers mainly through intermolecular hydrogen bonding on $\mathrm{NH}$ and $\mathrm{C}=\mathrm{O}$ groups. ${ }^{8}$ Importance of the carbamate residue for chiral recognition has recently been supported by the ${ }^{1} \mathrm{H}$ NMR study using cellulose tris(4-trimethylsilylphenylcarbamate). ${ }^{13}$ Moreover, it has also been proposed that intramolecular hydrogen bond between adjacent carbamate moieties of neighboring glucose units may be important for effective chiral recognition. ${ }^{11}$ Such an intramolecular hydrogen bond may contribute 

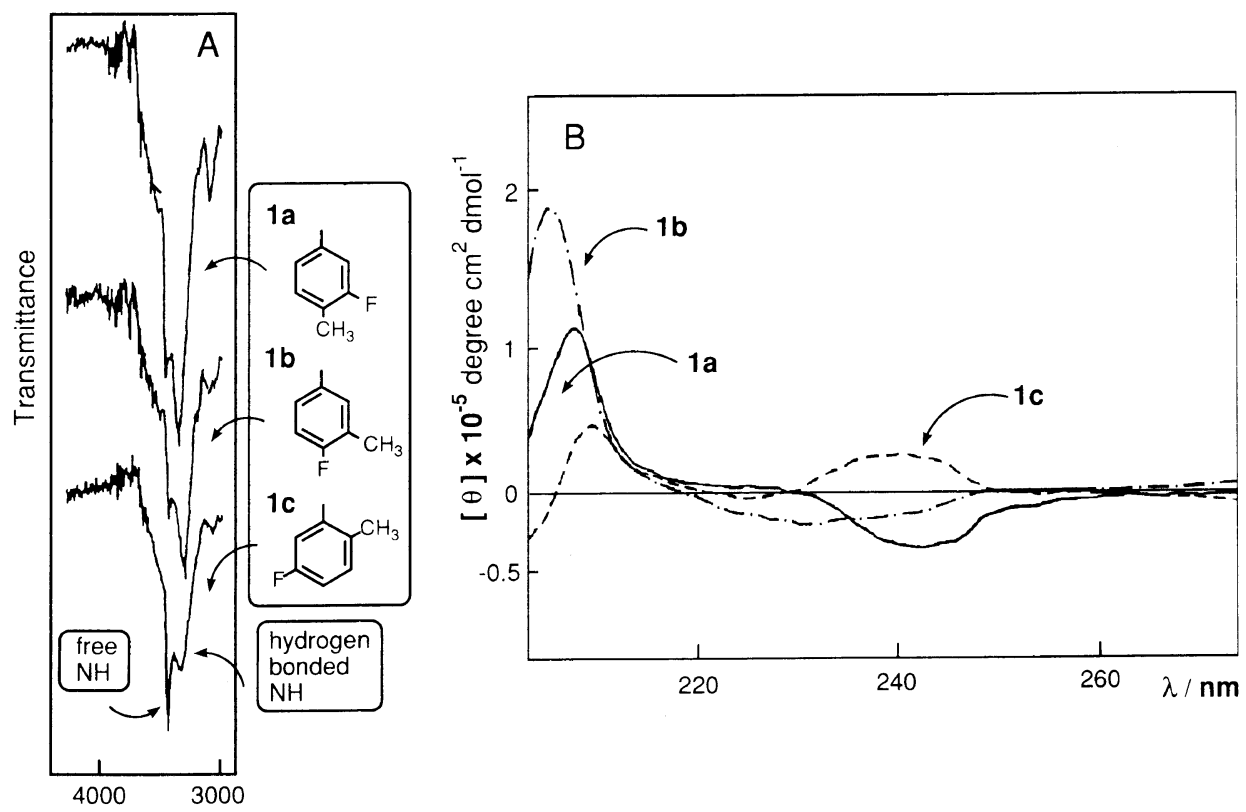

Wave number $/ \mathrm{cm}^{-1}$

Figure 4. IR (A) and CD (B) spectra of fluoro-methylphenylcarbamates of cellulose (1a, 1b, and 1c). CD spectra were measured in tetrahydrofuran (THF) $(1.6 \mathrm{mM})$ at ambient temperature.

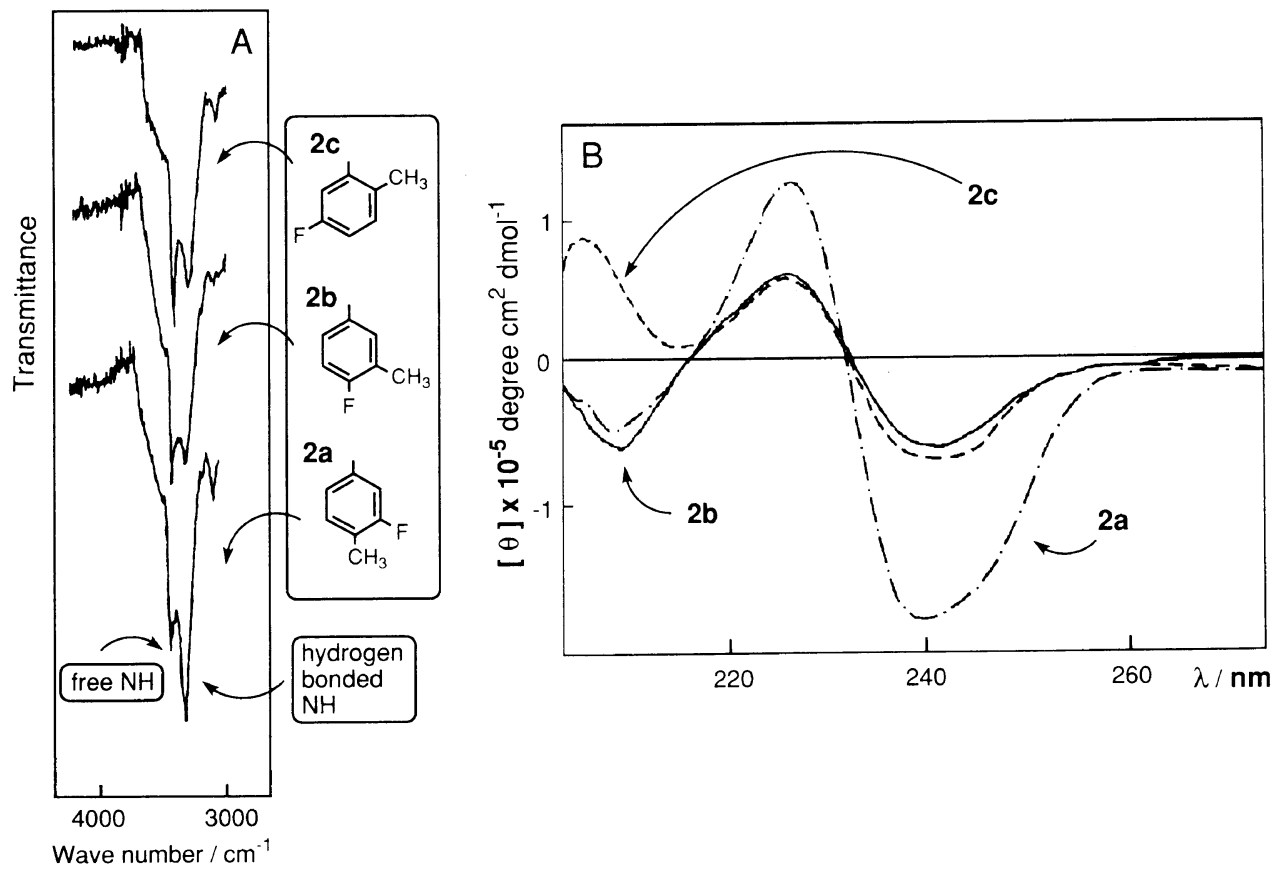

Figure 5. IR (A) and CD (B) spectra of fluoro-methylphenylcarbamates of amylose (2a, $\mathbf{2 b}$, and $\mathbf{2 c}$ ). CD spectra were measured in THF $(1.6 \mathrm{mM})$ at ambient temperature. 
to maintain a rigid regular structure of the phenylcarbamates of the polysaccharides.

To confirm this, IR and CD spectra were measured (Figures 4 and 5). Figure 4(A) shows IR spectra of the $\mathrm{N}-\mathrm{H}$ region of the fluoro-methylphenylcarbamates of cellulose. Two peaks are observed; the peak in the lower wave number is assigned to a $\mathrm{N}-\mathrm{H}$ group involved in intramolecular hydrogen bond between the carbamate residues of neighboring glucose units and the peak in the higher wave number to a free $\mathrm{N}-\mathrm{H}$ bond. ${ }^{9}$ Recent results of molecular mechanics calculation for cellulose trisphenylcarbamate showed the existence of intramolecular hydrogen bond. ${ }^{14}$ The derivatives $\mathbf{1 a}$ and $\mathbf{1 b}$ showing high chiral recognition have higher fraction of intramolecularly hydrogen bonded $\mathrm{N}-\mathrm{H}$ than the orthoand meta-disubstituted derivative 1c showing low chiral recognition. The former derivatives may have a rigid regular structure due to intramolecular hydrogen bonding which must be responsible for high chiral recognition. An evidence for the regular structure of $\mathbf{1 a}$ and $\mathbf{1 b}$ can be seen in the CD spectra (Figure 4(B)) which showed different peak top wavelengths and intensities depending on the position of the substituents, although the derivatives showed a similar UV spectral pattern. The CD signal at around $230-240 \mathrm{~nm}$ may be due to the carbamoyl groups and that in the region of $200-220 \mathrm{~nm}$ may be assigned to absorption mainly due to the phenyl groups, because aliphatic carbamate derivatives of cellulose such as cellulose tris(2-propylcarbamate) showed a very weak absorption in the region of $200-220 \mathrm{~nm}^{15}$ The derivatives $\mathbf{1 a}$ and $\mathbf{1 b}$ showed a more intense CD signal than $1 c$ in this region. These results suggest that $\mathbf{1 a}$ and 1b may have highly regular structures which lead to high chiral recognition.

The IR and CD spectra of the amylose derivatives are shown in Figure 5. Contrary to the cellulose derivatives, ortho-substituted $\mathbf{2 c}$ contained a higher fraction of $\mathrm{N}-\mathrm{H}$ involved in intramolecular hydrogen bond than $\mathbf{2 a}$ and 2b (Figure 5(A)). The CD spectrum of the ortho-substituted derivative showed a different pattern of $C D$ with a more intense signal in the region of $200-220 \mathrm{~nm}$ (Figure $5(\mathrm{~B})$ ). These amylose derivatives may have different ordered structures depending on the position of the substituents. The different order structures may result in characteristic high chiral recognition ability of each derivative.

Acknowledgments. This work was partially supported by Grant-in-Aids for Scientific Research No. 05559009 and on Priority Areas No. 06242101 from the Ministry of Education, Science, and Culture of Japan.

\section{REFERENCES}

1. S. Ahuja, in "Chiral Separation by Liquid Chromatography (ACS symposium Series, No. 471)," American Chemical Society, Washington, D.C., 1991.

2. W. H. Pirkle and T. C. Pochapsky, Chem. Rev., 89, 347 (1989).

3. D. R. Taylor and K. Maher, J. Chromatogr. Sci., 30, 67 (1992).

4. S. G. Allenmark, in "Chromatographic Enantioseparation," Ellis Horwood, Chichester, 1988, Chapter 7, p 90.

5. Y. Okamoto and Y. Kaida, J. Chromatogr. A., 666, 403 (1994).

6. A. Ishikawa and T. Shibata, J. Liq. Chromatogr., 16, 859 (1993).

7. J. Dingenen, in "A Practical Approach to Chiral Separations by Liquid Chromatography," G. Subramanian, Ed., VCH Publishers, New York, 1994, Chapter 6, p 115.

8. Y. Okamoto, M. Kawashima, and K. Hatada, J. Chromatogr., 363, 173 (1986).

9. B. Chankvetadze, E. Yashima, and Y. Okamoto, $J$. Chromatogr. A, 670, 39 (1994).

10. B. Chankvetadze, E. Yashima, and Y. Okamoto, Chem. Lett., 617 (1993).

11. B. Chankvetadze, E. Yashima, and Y. Okamoto, $J$. Chromatogr. A, 694, 101 (1995).

12. Y. Kaida and Y. Okamoto, Bull. Chem. Soc. Jpn., 65, 2286 (1992).

13. E. Yashima, M. Yamada, and Y. Okamoto, Chem. Lett., 579 (1994).

14. E. Yashima, M. Yamada, Y. Kaida, and Y. Okamoto, J. Chromatogr. A, 694, 347 (1995).

15. Y. Okamoto and Y. Kaida, unpublished results. 